www.jmscr.igmpublication.org

Impact Factor 5.84

Index Copernicus Value: 83.27

ISSN (e)-2347-176x ISSN (p) 2455-0450

crossref DOI: https://dx.doi.org/10.18535/jmscr/v5i2.01

Journal Of Medical Science And Clinical Research

\title{
Prevalence of Lower Limb Traction Apophysitis among Children with Low Vitamin D and Secondary Hyperparathyroidism - Ultra Sonographic Study
}

\author{
Authors \\ Ahmed Fathy Abogamal', Eman Mahmoud ${ }^{2}$, Ahmed Aly Abdelaziz ${ }^{3}$, Sameh Abdelatif $^{4}$, \\ Mohammed Ahmed HasunaHemeda ${ }^{5}$, Ahmed FathyAbdalaziz ${ }^{6}$, Maged A H Shoukeer ${ }^{7}$ \\ ${ }^{1,3}$ Assistant Professor of Rheumatology \& Rehabilitation, Faculty of Medicine, AL-Azhar University Cairo- \\ Egypt \\ ${ }^{2}$ Lecturer of Endocrinology, Faculty of Medicine for Girls, AL-Azhar University Cairo- Egypt \\ ${ }^{4}$ Lecturer of rheumatology\& Rehabilitation, Faculty of Medicine, AL-Azhar University Cairo- Egypt \\ ${ }^{5}$ Lecturer of medicine, faculty of medicine, Zagazig University, Zagazig - Egypt \\ ${ }^{6}$ Assistant Professor of Clinical Pathology, Faculty of Medicine, Al-Azhar University, Cairo, Egypt \\ ${ }^{7}$ Assistant Professor of Radiology, Faculty of Medicine, Al-Azhar University, Cairo, Egypt \\ Corresponding Author
}

Ahmed Fathy Abogamal

94 Aiadwasef, Ahmed Esmat Street, Ain Shams 11311 Cairo-Egypt

Email: ahmedfathy15@yahoo.com,Tel:00201001972099

\begin{abstract}
Introduction: Traction apophysitis is a traction phenomenon resulting from repetitive load through the tendon or ligament at its insertion upon the skeletally immature bony insertion. Secondary hyperparathyroidism along with vitamin $D$ deficiency have a major deleterious effect on the musculoskeletal system and may affect the traction points on the children age group.

Aim of the work: Is to investigate the prevalence of lower limb traction apophysitis among children with secondary hyperparathyroidism and low vitamin $D$.

Patients and Methods: Group I: 68 patients with Secondary hyperparathyroidism due to hypovitaminosis D; Group II: 50 normal children as a control group. All patients were subjected to clinical history taking, examination for the traction apophysitis sites in the lower limb, body mass index measurement, ultrasound assessment of Quadriceps tendon insertion, Infrapatellar ligament, Achilles tendon, and Plantar fascia.

Results: The ultrasound detected prevalence of traction apophysitis in the lower limb in the study group was $66.2 \%$, were 45 patients defined affected, among the affected patients 32 (71.1\%) were male and 13(28.9\%) were female. Ultrasound assessment of the control group shows that $10(20 \%)$ patients affected by traction apophysitis; among the affected patients $8(80 \%)$ were male and $2(20 \%)$ were female.

Conclusion: Traction apophysitis appears to be frequent problem in children with secondary hyperparathyroidism and low vitamin D. The factors significantly associated with presence of traction apophysitis were increased parathyroid hormone and low vitamin $D$.

Keywords: traction apophysitis, secondary hyperparathyroidism, low vitamin D.
\end{abstract}




\section{Introduction}

Secondary hyperparathyroidism refers to the increased excessive secretion of parathyroid hormone (PTH) in a protective feedback process from our body against low serum calcium level to maintain calcium homeostasis. Another essential player in the regulation of calcium and phosphorus balance is the Vitamin D. Its main function is to increase the availability of calcium and phosphorus essential for the homeostasis. Long standing deficiency of vitamin D disturbs the arms of calcium homeostasis with a resultant secondary hyperparathyroidism as a trial form the body to resolve this disturbance. ${ }^{(1,2)}$

Secondary hyperparathyroidism along with vitamin $\mathrm{D}$ deficiency have a major deleterious effect on the musculoskeletal system especially on the children age group. Bone metabolism in this age group is significantly affected by a resultant bony pains, osteomalacia, deformities, and even green stick fractures. ${ }^{(1,2)}$

A part from the musculoskeletal system is the anchoring tendons and ligaments insertions, which are susceptible for much traction load and their attachment to bone usually came through fibro cartilaginous insertions. Due to its anatomical, and physiological properties these traction points are responsible for a significant percent of joint pain especially in children and adolescent age group due to traction apophysitis. ${ }^{(3-6)}$

Traction apophysitis is a traction phenomenon resulting from repetitive load through the tendon or ligament at its insertion upon the skeletally immature bony insertion. This occurs in preadolescence age; commonly at the tibial tubercle, quadriceps - patella, the patella-patellar tendon junction, Achilles tendon insertion, and the plantar fascia attachment to calcaneus. ${ }^{(4-6)}$

Assessment of traction points by conventional $\mathrm{X}$ ray usually misses higher sensitivity due to the unreliability of $\mathrm{X}$ ray to assess the soft tissue component at insertion points. Ultrasound can effectively assess the insertion points, tendons, ligaments, cartilage and the bone cortex as well. ${ }^{(7)}$
As the secondary hyperparathyroidism and vitamin $\mathrm{D}$ deficiency have a significant effect on skeletal metabolism and maturity; which in turn affect bony component of the traction tendons and ligament insertions, we conducted this research to investigate these insertion points in such patients depending on the reliability and sensitivity of ultrasound in detection of prevalence of traction apophysitis in children.

\section{Aim of the work}

Is to investigate the prevalence of lower limb traction apophysitis among children with seconddary hyperparathyroidism and low vitamin D.

\section{Patients and Methods}

Study design \& setting: The current study was based on a cross sectional hospital-based survey, conducted among children with secondary hyperparathyroidism presenting at outpatient rheumatology and endocrinology clinics of ALAZHAR University Hospitals, Egypt through 2016.

\section{Inclusion Criteria}

- Children age 10-16 years old.

- Secondary hyperparathyroidism.

- Hypovitaminosis D.

\section{Exclusion Criteria}

- Recent lower limb trauma or intra-articular procedures.

- Previous lower limb surgery.

- Patients with chronic systemic diseases that could affect patient; e.g. renal, liver, cardiac impairment, thyroid dysfunction, or neurological disorder.

- Inflammatory joint diseases, as rheumatoid arthritis, crystal arthritis, and spondyloarthropathy.

- Drugs that could affect parathormone, tendons or bone metabolism

\section{Study Sample}

- Al-Azhar University Hospitals contain 2 outpatient rheumatology clinics (AlHussein University hospital clinic and ElSayed Galal University hospital clinic); and one endocrinology clinic. The three 
clinics were included in the study. All children with Secondary hyperparathyroidism and Hypovitaminosis D were asked to be included in the study where only 68 patients accepted to participate. Oral consent was obtained from every participant before taking part in the study with nearly $95 \%$ response rate

\section{Data Collection}

The data was collected through an interview questionnaire from all participants. The questionnaire used in the study was prepared by the researcher himself and included questions on demographic factors, causes, as well as questions on risk factors associated with the presence of traction apophysitis. Field survey was conducted after obtaining approval from hospital authority and Research Ethical Committee. The data was collected throughout 2016. The field work took 1 days/week for every clinic. The average number of children interviewed per day was 1-2 and the time needed to complete the questionnaire was 912 minutes.

\section{Grouping:}

Group I: 68 patients with Secondary hyperparathyroidism due to hypovitaminosis D.

Group II: 50 normal children as a control group.

All patients were subjected to clinical history taking, examination for the traction apophysitis sites in the lower limb, and body mass index measurement.

Ultrasonography: was performed using a scanner with a multi-frequency $12 \mathrm{~L}$ linear array transducer (General electric Systems; LOGIQU-E). Ultrasonography (US) has been used to detect features of apophysitis, including: Cartilage swelling, Fragmentation, tendon lesions, and related bursitis. ${ }^{(7-10)}$ Ultrasound techniques were used for all patients included in the study for the following regions:

- Quadriceps tendon insertion: at the superior pole of the patella, assessed in long and short axis with the patient knee flexed 45 degree.

- Infrapatellar ligament: at its proximal attachment to the inferior pole of the patella, and distally at tibial tuberosity assessed in long and short axis with the patient knee flexed 45 degree.

- Achilles tendon: at the posterior surface of the calcaneus with patient prone, foot hanging from the examination table, and the ankle in 90 degree, assessed in long and short axis.

- Plantar fascia: at the inferior surface of the calcaneus with patient prone, foot hanging from the examination table, and the ankle in 90 degree, assessed in long and short axis. ${ }^{(7)}$

\section{Laboratory assessment:}

Sampling: $8 \mathrm{ml}$ of venous blood was collected by venipuncture under complete aseptic technique from each subject in the study (After obtaining the Research/Ethics Committee approval and written informed consents) and divided as follow

1- One $\mathrm{ml}$ blood in EDTA tube for CBC

2- $1.6 \mathrm{ml}$ of blood on citrated tube for ESR

3 - the remaining $5 \mathrm{ml}$ is divided into 2 portions the first is rapidly centrifuged and the resulting serum separated and divided into 2 aliquots, one aliquot for immediate determination of PTH while the other aliquot was kept frozen at-20C until used for assay of vitamin $\mathrm{D}$. The 2 nd portion left for 30 minutes until clotted and the separated serum was used for determination of routine biochemical laboratory investigations (including liver functions, Kidney functions and Random blood sugar), Total Calcium, inorganic phosphorous and ALP.

\section{Methods}

1- CBC was done by Sysmex Kx- 21N (fully automated cell counter). (Roche diagnostics).

2- First hour ESR by Westergren method.

3- Total Calcium, inorganic phosphorous, ALP and routine biochemical laboratory investigations (including liver functions, Kidney functions and Random blood sugar), all were assayed by fully automated Chemistry analyzer Biolis 50i Superior With normal ranges; Total serum calcium (8.810.8) $\mathrm{mg} / \mathrm{dL}$, Serum phosphorus (4.0-7.0)mg/dL, Alkaline phosphatase 180-450 u/L. 
4- Assay of PTH hormone by chemiluminiscence method using cobas e 411 analyzer (Roche diagnostics) normal range: PTH (10-65)pg/mL.

5- Serum $25(\mathrm{OH})$ vitamin $\mathrm{D}$ : using commercially available (ELISA) Kit supplied by Calbiotech's, Inc, USA.*

\section{Principle}

The Calbiotech Vitamin D Kit is a solid phase enzyme-linked immunoassay (ELISA), based on the principal of competitive binding. Anti-Vitamin D antibody coated wells are incubated with Vitamin D standards, controls, samples, and vitamin D-Biotin conjugate at room temperature for 90 minutes. During the incubation, a fixed amount of biotin-labeled vitamin D competes with the endogenous Vitamin D in the sample, standard, or quality control serum for a fixed number of binding sites on the anti Vitamin D antibody. Following a wash step, bound Vitamin D-Biotin is detected with Streptavidin-HRP. Streptavidin-HRP conjugate immunologically bound to the well progressively decreases as the concentration of Vitamin D in the specimen increases. Unbound SA-HRP conjugate is then removed and the wells are washed. Next, a solution of TMB Reagent is added and incubated at room temperature for 30 minutes, resulting in the development of blue color. The color development is stopped with the addition of stop solution, and the absorbance is measured spectrophotometrically at $450 \mathrm{~nm}$. A standard curve is obtained by plotting the concentration of the standard versus the absorbance. The color intensity will be inversely proportional the amount of $25(\mathrm{OH}) \mathrm{D}$ in the sample. The total assay procedure run time is 2.5 hours.

* Address: Stree Street: 10461 Austin Drive City: Spring Valley State: CA

ZIP Code: 91978 Toll-Free :( 866) CALBIOTECH Local :) 619) 660-6162 Fax :( 619) 660-6970

E-Mail: info@calbiotech.com The ranges of vitamin D were: 1) sufficiency: equal or more than 30ng/ml, 2) insufficiency: from 10-30ng/ml, and 3) deficiency: less than $10 \mathrm{ng} / \mathrm{ml} .^{(11)}$

Data analysis: Data were entered, organized, tabulated and analyzed using the standard computer program SPSS version 19. Quantitative data were expressed as Mean $\pm \mathrm{SD}$, while qualitative data were expressed as frequency and percent. Student t-test was used to measure the difference between means of two quantitative groups, with the significant level set at 0.05 , and highly significant at 0.01 . Chi square test for comparison of qualitative data.

\section{Results}

Descriptive analysis group I, shows that age was 13.25 \pm 1.51 , BMI $23.15 \pm 1.98$, PTH $340.97 \pm$ 149.43 , vitamin D $5.97 \pm 2.25$, alkaline phosphatase $463.03+152.41$, serum calcium $7.93 \pm 0.35$ and phosphorus 3.70 \pm 0.54 .Table (1), figure (1) While group II age was $13.36 \pm 1.51$, BMI $23.06 \pm 1.98, \quad$ PTH $40.38 \pm 12.47$, vitamin D $33.56 \pm 6.54$, alkaline phosphatase $125.92 \pm 6.54$, serum calcium $9.32 \pm 0.45$ and phosphorus $3.62 \pm 0.45$. Table (1), figure (1)

As regards to sex distribution in group I males were 38 (55.9\%), and females were $30(44.1 \%)$; while in group II males were $28(56 \%)$, and females were 22 (44\%). There was no significant difference as regard the sex distribution as the chisquare statistic was 0.99 , not significant at $\mathrm{p}<0.05$. The ultrasound detected prevalence of traction apophysitis in the lower limb in the study group was $66.2 \%$, were 45 patients defined affected, among them $32(71.1 \%)$ were males and $13(28.9 \%)$ were females. So the boys were significantly affected that girls as the chi-square statistic was12.52 and the result were significant at $\mathrm{p}<0.05$.Table (2), figure (2)

The quadriceps tendon insertion was affected on right side in $4(5.9 \%)$ patients, left side in $3(4.4 \%)$ patients and no cases affected bilaterally. Proximal attachment of infrapatellar ligament to inferior pole of the patella found affected on the right side in $8(11.8 \%)$ patients, left side in 11 $(16.2 \%)$ patients and no cases affected bilaterally. 
distal attachment of infrapatellar ligament to tibial tuberosity found affected on the right side in 5 $(7.6 \%)$ patients, left side in $6(8.8 \%)$ patients and $16(23.5 \%)$ cases affected bilaterally.

Insertion of the Achilles tendon to the posterior surface of the calcaneus was affected on right side in $3(4.4 \%)$ patients, left side in $1(1.5 \%)$ patients and $2(2.9 \%)$ cases affected bilaterally. As regards to theplantar fascia insertion to the inferior surface of the calcaneusfound affected on the right side in $4(5.9 \%)$ patients, left side in $8(11.8 \%)$ patients and $5(7.4 \%)$ cases affected bilaterally. Table (3), figure $(3,4,5)$

Ultrasound assessment of the control group shows that $10(20 \%)$ patients affected by traction apophysitis; among the affected patients $8(80 \%)$ were males and 2 (20\%) were females. Table (3), figure $(3,4,5)$

The right quadriceps tendon insertion in $2(2.9 \%)$ cases, distal attachment of infrapatellar ligament to tibial tuberosity $1(1.5 \%)$ case right, $4(5.9 \%)$ on the left side; while plantar fascia affected in $1(1.5 \%)$ case right, $4(2.9 \%)$ on the left side. Table (3), figure $(3,4,5)$

TA was significantly higher in the study group the control group as the chi-square statistic was 23.85 and the result were significant at $\mathrm{p}<0.05$.

Comparing means of both groups shows no statistical significant difference as regards to the age, BMI, and phosphorus. While there were statistical significant difference between both groups in the $\mathrm{PTH}$, vitamin $\mathrm{D}$, calcium, and alkaline phosphatase.Table (1), figure (1)

Table (1): Descriptive results of both groups

\begin{tabular}{|l|c|c|c|c|c|c|}
\hline \multirow{2}{*}{} & \multicolumn{2}{|c|}{ Group I } & \multicolumn{2}{c|}{ Group II } & \multicolumn{2}{c|}{ Comparison } \\
\cline { 2 - 7 } & Mean & $\begin{array}{c}\text { Std. } \\
\text { Deviation }\end{array}$ & Mean & $\begin{array}{c}\text { Std. } \\
\text { Deviation }\end{array}$ & $\mathrm{P}$ & significance \\
\hline AGE years & 13.25 & 1.51 & 13.36 & 1.51 & $>0.05$ & $\mathrm{NS}$ \\
\hline BMI & 23.15 & 1.98 & 23.06 & 1.98 & $>0.05$ & $\mathrm{NS}$ \\
\hline PTH pg/mL & 340.97 & 149.43 & 40.38 & 12.47 & $<0.05$ & $\mathrm{~S}$ \\
\hline VITD ng/ml & 5.97 & 2.25 & 33.56 & 6.54 & $<0.05$ & $\mathrm{~S}$ \\
\hline ALP U/L & 463.03 & 152.41 & 125.92 & 6.54 & $<0.05$ & $\mathrm{~S}$ \\
\hline $\mathrm{Ca} \mathrm{mg/dL}$ & 7.93 & 0.35 & 9.32 & 0.45 & $<0.05$ & $\mathrm{~S}$ \\
\hline Ph mg/dL & 3.70 & 0.54 & 3.62 & 0.45 & $>0.05$ & $\mathrm{NS}$ \\
\hline
\end{tabular}

(BMI: body mass index; PTH: parathormone; VITD: vitamin D; ALP: alkaline phosphatase; Ca: Calcium; Ph: phaosphorus; NS: non-significant; S: significant)

Table (2): sex distribution among groups.

\begin{tabular}{|c|l|c|c|c|c|}
\hline \multicolumn{2}{|c|}{} & \multicolumn{2}{c|}{ Male } & \multicolumn{2}{c|}{ Female } \\
\cline { 3 - 6 } \multicolumn{2}{|c|}{} & $\mathrm{N}$ & $\%$ & $\mathrm{~N}$ & $\%$ \\
\hline \multirow{3}{*}{ GI } & Total & 38 & 55.9 & 30 & 44.1 \\
\cline { 2 - 6 } & Affected & 32 & 71.1 & 13 & 28.9 \\
\hline \multirow{3}{*}{ GII } & Total & 28 & 56 & 22 & 44 \\
\cline { 2 - 6 } & Affected & 8 & 80 & 2 & 20 \\
\hline
\end{tabular}

Table (3): ultrasound detected traction apophysitis in both groups.

\begin{tabular}{|c|c|c|c|c|c|c|c|c|c|c|c|c|c|c|c|c|c|}
\hline & \multicolumn{3}{|c|}{$\begin{array}{l}\text { Quadriceps } \\
\text { insertion }\end{array}$} & \multicolumn{3}{|c|}{$\begin{array}{l}\text { Inferior patellar } \\
\text { pole }\end{array}$} & \multicolumn{3}{|c|}{ Tibial tuberosity } & \multicolumn{3}{|c|}{$\begin{array}{l}\text { Achilles } \\
\text { insertion }\end{array}$} & \multicolumn{3}{|c|}{ Plantar fascia } & \multirow{2}{*}{$\begin{array}{c}\text { Total } \\
\text { affected }\end{array}$} \\
\hline & & $\mathrm{Rt}$ & $\mathrm{Lt}$ & $\mathrm{Bi}$ & $\mathrm{Rt}$ & $\mathrm{Lt}$ & $\mathrm{Bi}$ & $\mathrm{Rt}$ & $\mathrm{Lt}$ & $\mathrm{Bi}$ & $\mathrm{Rt}$ & $\mathrm{Lt}$ & $\mathrm{Bi}$ & $\mathrm{Rt}$ & $\mathrm{Lt}$ & $\mathrm{Bi}$ & \\
\hline \multirow{2}{*}{ GI } & $\mathrm{N}$ & 4 & 3 & 0 & 8 & 11 & 0 & 5 & 6 & 16 & 3 & 1 & 2 & 4 & 8 & 5 & 45 \\
\hline & $\%$ & 5.9 & 4.4 & 0 & 11.8 & 16.2 & 0 & 7.6 & 8.8 & 23.5 & 4.4 & 1.5 & 2.9 & 5.9 & 11.8 & 7.4 & 66.2 \\
\hline \multirow{2}{*}{ G II } & $\mathrm{N}$ & 2 & 0 & 0 & 0 & 0 & 0 & 1 & 4 & 0 & 0 & 0 & 0 & 1 & 2 & 0 & 10 \\
\hline & $\%$ & 2.9 & 0 & 0 & 0 & 0 & 0 & 1.5 & 5.9 & 0 & 0 & 0 & 0 & 1.5 & 2.9 & 0 & 20 \\
\hline
\end{tabular}

(GI: Group I; GII: Group II; Rt: right; Lt: left; Bi: bilateral; N: number) 
Figure (1): descriptive statistics of both groups.

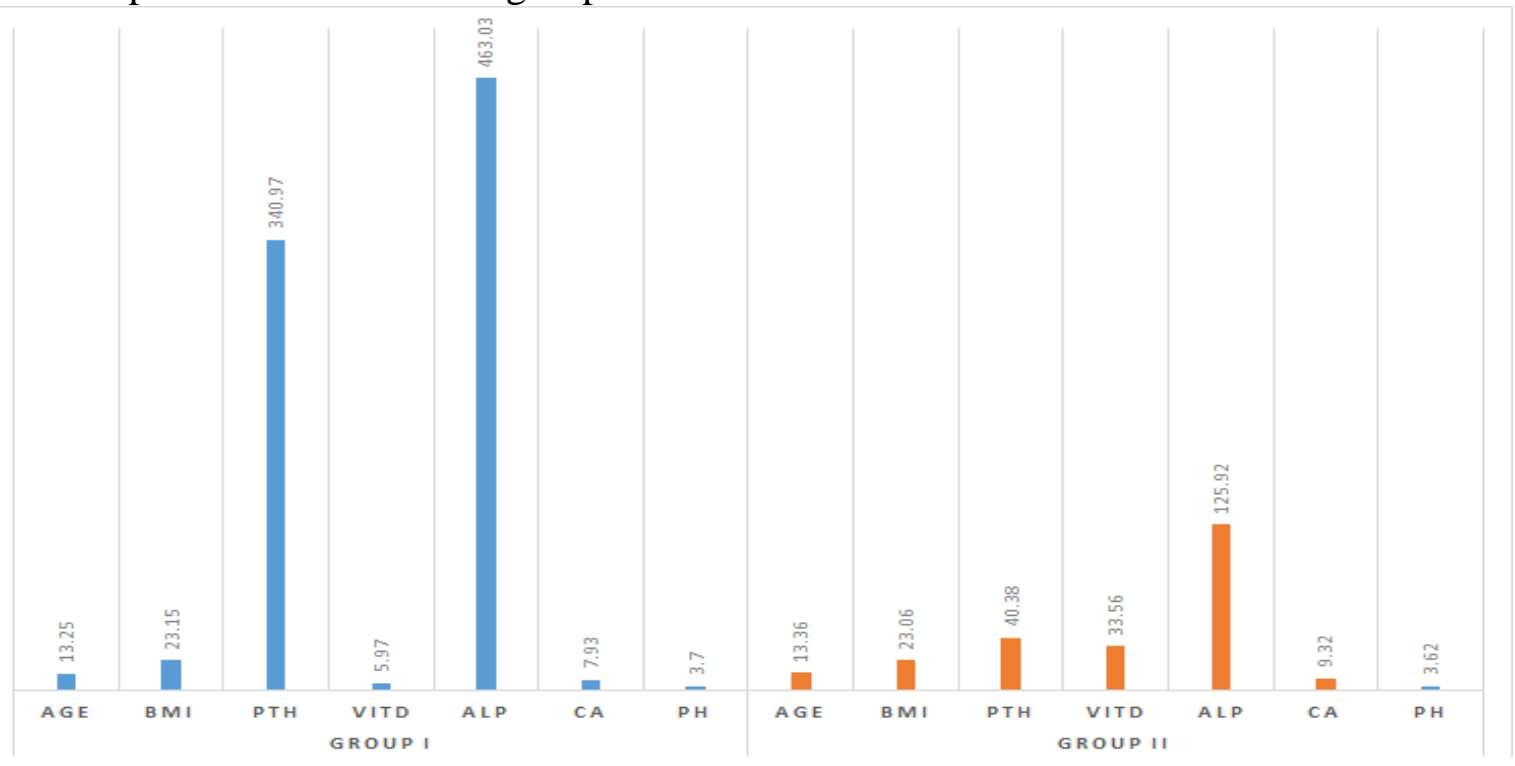

Figure (2): sex distribution among groups.

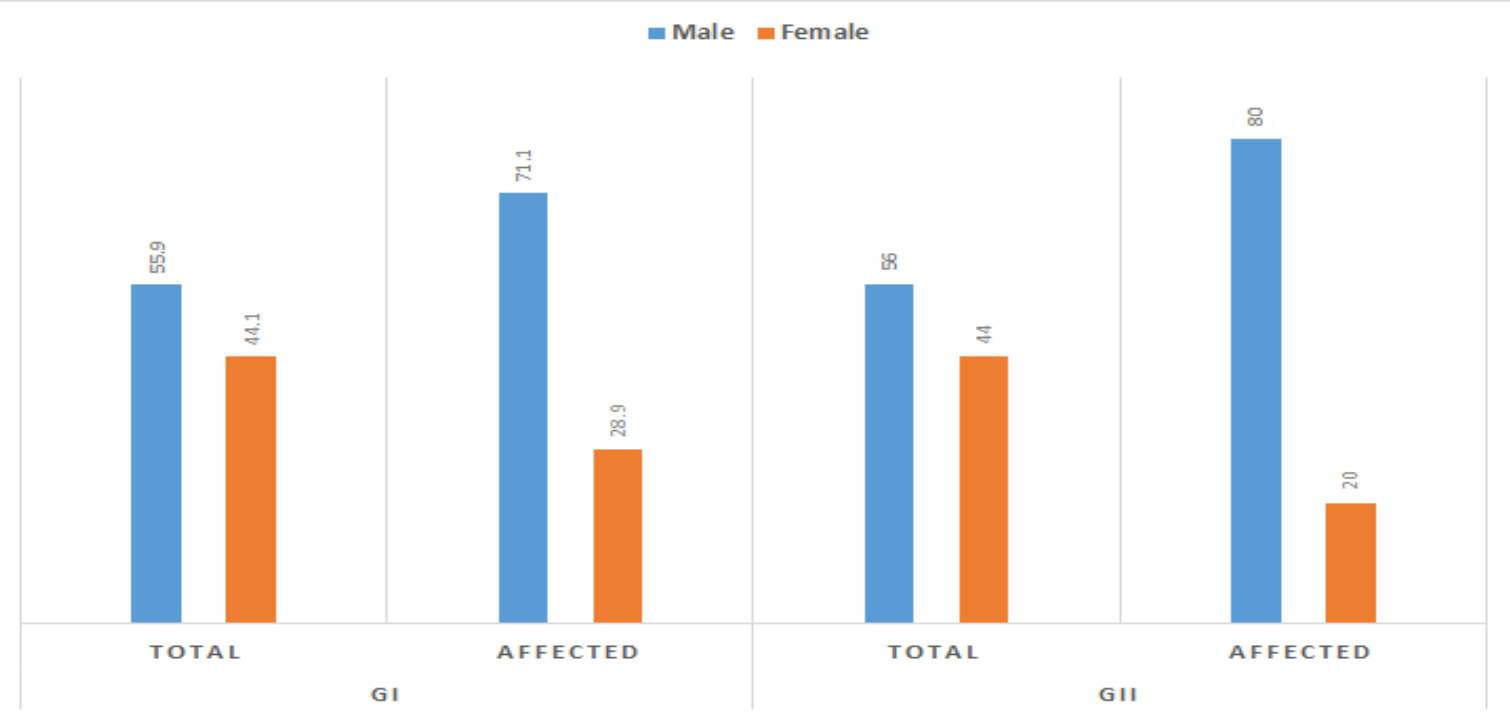

Figure (3): ultrasound finding of quadriceps - patella insertion, and patella - infrapatellar ligament insertion.

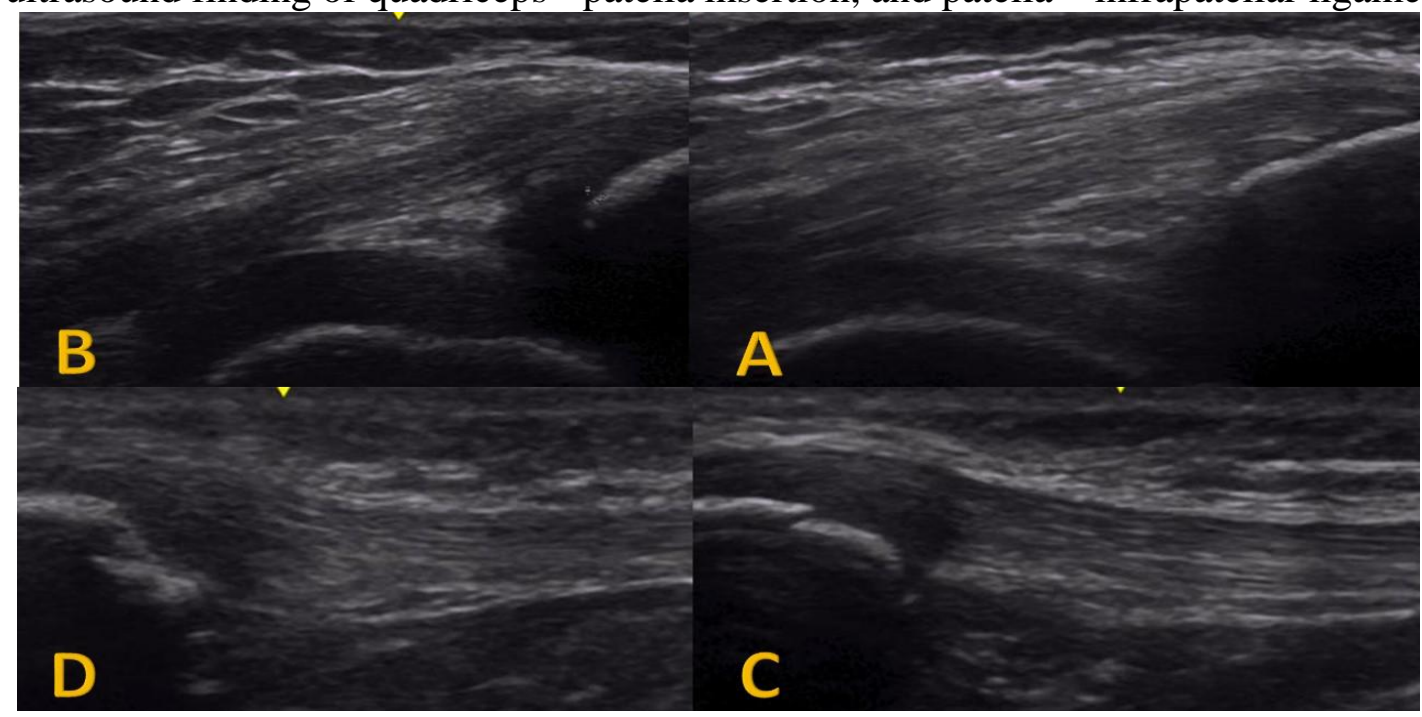

(A: normal quadriceps - patella insertion, B: traction apophysitis quadriceps - patella insertion; C: normal patella - infrapatellar ligament insertion, D: traction apophysitis patella - infrapatellar ligament insertion) 
Figure (4): ultrasound finding of infrapatellar ligament insertion to tibial tuberosity.

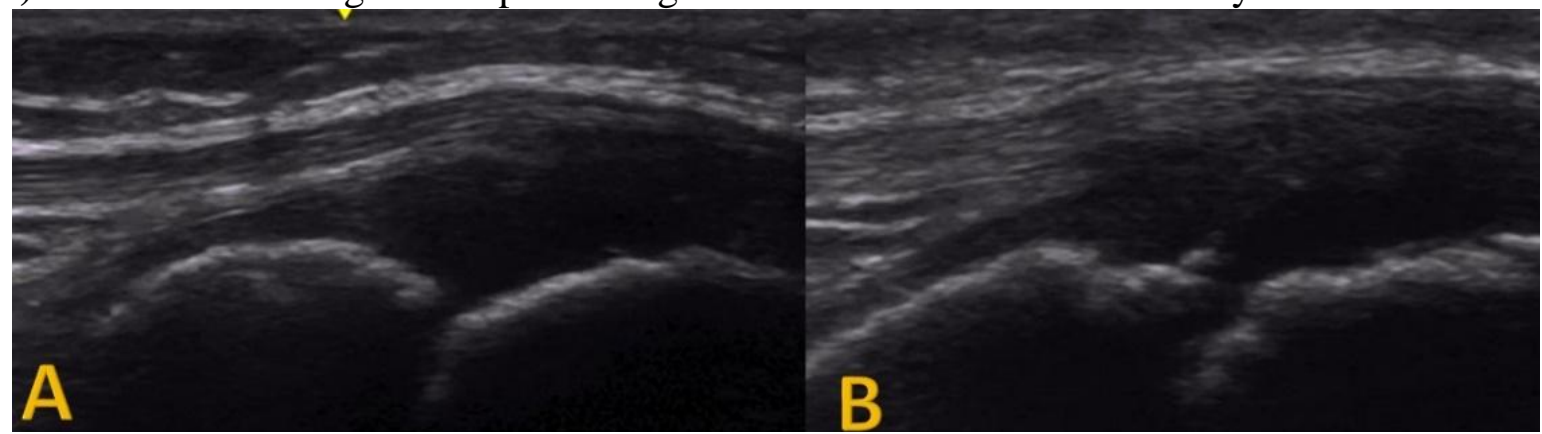

(A: normal infrapatellar ligament insertion to tibial tuberosity, B: traction apophysitis infrapatellar ligament insertion to tibial tuberosity)

Figure (5): ultrasound finding of achillis tendon insertion to posterior surface of the calcaneus, and plantar fascia insertion to inferior surface of the calcanus.

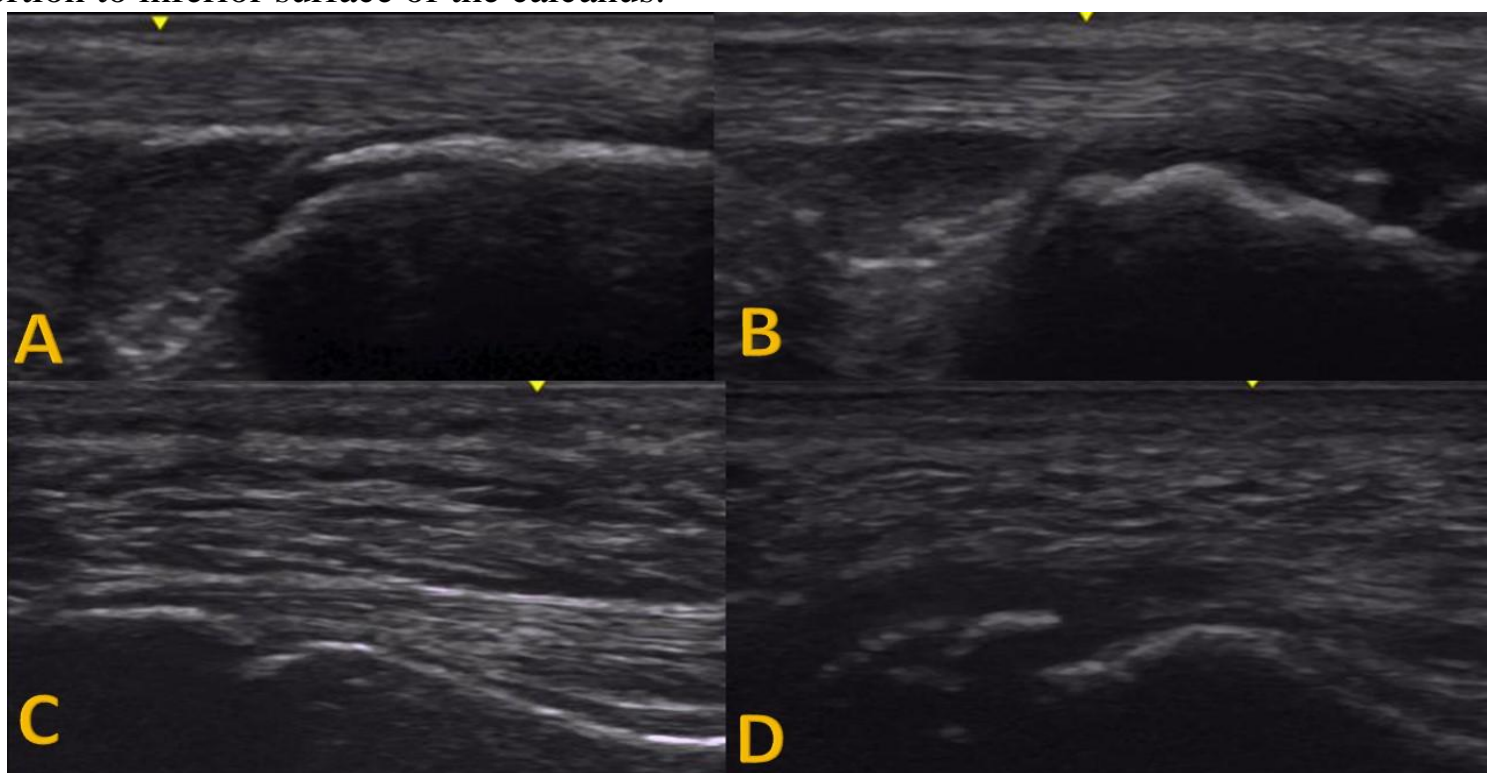

(A: normal achillis tendon insertion, B: traction apophysitis achillis tendon insertion; C: normal plantar fascia insertion, D: traction plantar fascia insertion)

\section{Discussion}

Traction apophysitis (TA) frequently encountered among growing children and represent a common cause of lower limb pain this age group with immature skeleton. Presence of hyperparathyroidism with low vitamin $\mathrm{D}$ in children with growing skeleton expected to significantly affect the immature skeleton with much pain and deformities. $^{(12,1,2)}$

In the current study we investigate the prevalence of traction apophysitis as frequent cause of lower limb pain in especial group of children with secondary hyperparathyroidism. According to our results the prevalence of TA was $66.2 \%$, where 45 patients out of 68 were affected, among the affected patients boys (71.1\%) significantly affected than girls $(28.9 \%)$. TA at the tibial tuberosity was the commonest among the patients studied, and usually presented bilaterally, followed by TA at the plantar fascia insertion, and the Achillis tendon. In comparison to the control group, with matched age, BMI, and sex distribution; only $20 \%$ have been found affected by TA, which represent a significant impact for the hyperparathyroidism and low vitamin $\mathrm{D}$, on the traction points among children.

The reported higher prevalence of TA among boys than girls may be attributed for the higher activity and the repeated traction injury to the tendon and ligament insertion, which has been reported in many other similar studies about TA which support our results. ${ }^{(13-16)}$ 
Traction points studied were either tendons or ligaments inserted to bone cortex through transitional interface called the enthesis. These enthesis histologically represent a transition from tendon or ligament side to bony side with different thickness depending on its location, with gradient increased mineralization in the direction towards the bone. According to our knowledge all previous studies about TA describes its occurrence due to repeated traction trauma on the immature skeleton, without concern about the bone metabolism in such cases either is it normal or abnormal. There is no doubt that Parathyroid hormone and vitamin $\mathrm{D}$, plays an essential role in bone development, mineralization, and maturation, so with their disturbance the resultant delayed bony maturation with deficient mineralization has been supposed to affect the enthesis traction points. ${ }^{(17-}$ 19)

With weakness of the enthesis traction points and the load applied from the repeated traction there will be an increased susceptibility to the development of TA, which could be responsible for the high prevalence of TA among our patients and represents hyperparathyroidism and low vitamin $\mathrm{D}$ as an important risk factors for the development of TA.

With the development of ultrasound (US) high frequency probes, and its introduction in the musculoskeletal field; US has been shown to be very reliable and sensitive tool for assessing many articular and periarticular lesions. In the current study US simply defined the tendon and ligament insertions with real time imaging and at low cost in comparison to MRI. ${ }^{(7,20)}$

Our study limitation includes lack of MRI studies for the traction points, but it will be very expensive to add MRI study for all these areas in such number of patients, plus the significant low cost, reliability and sensitivity of US which has been proven in musculoskeletal field which made our decision to rely on US only in our study.

Another important limitation was the lack of histopathological assessment for the traction points to examine the mineralization and bone maturation at the tissue level in both normal and affected children; which could explain the actual role of parathyroid hormone and vitamin D in TA. So we suggest application of histopathological studies for traction points in suitable animal models.

We suggest also an ultrasonographic follow up study for such patients with sufficient vitamin D supplementation to correct the secondary hyperparathyroidism to evaluate the effect of low vitamin D treatment on TA.

According to our results we recommend assessment of parathyroid hormone, and vitamin $\mathrm{D}$ in all children with TA, and US assessment of traction points in patients with hyperparathyroidism and low vitamin $\mathrm{D}$.

\section{Conclusion}

Traction apophysitis appears to be frequent problem in children with secondary hyperparathyroidism and low vitamin D. The factors significantly associated with presence of traction apophysitis were increased parathyroid hormone and low vitamin $\mathrm{D}$.

\section{Acknowledgment}

We would like to thank all patients who shared in the study, the authorities in Al-Azhar University Hospitals and everyone who helped us to accomplish this work.

\section{References}

1. Marx SJ: Hyperparathyroid and hypoparathyroid disorders. $N$ Engl $J$ Med 2000; 343:1863-1875.

2. Potts JT: Parathyroid hormone: Past and present. J Endocrinol 2006; 188:135-153

3. $\mathrm{Lu} \mathrm{HH}$, Thomopoulos S. Functional attachment of soft tissues to bone: development, healing, and tissue engineering. Annu Rev Biomed Eng. 2013;15:201-226.

4. Pommering TL, Kluchurosky L. Overuse injuries in adolescents. Adolesc Med State Art Rev. 2007 May. 18(1):95-120, ix. 
5. Krause BL, Williams JP, Catterall A. Natural history of Osgood-Schlatter disease. J PediatrOrthop. 1990 Jan-Feb. 10(1):65-8.

6. Weiss JM, Jordan SS, Andersen JS, Lee BM, Kocher M. Surgical treatment of unres-olved Osgood-Schlatter disease: ossicle resection with tibialtubercleplasty. J Pediatr Orthop. 2007 Oct-Nov. 27(7):844-7.

7. Bianchi S, Martinoli C: Ultrasound of the Musculoskeletal System. Springer -Verlag, Berlin, Heidelberg 2007.

8. Blankstein A, Cohen I, Heim M, et al. Ultrasonography as a diagnostic modality in Osgood-Schlatter disease. A clinical study and review of the literature. Arch Orthop Trauma Surg. 2001;121:536-539.

9. Ducher G, Cook J, Spurrier D, Coombs P, Black J, Bass S. Ultrasound imaging of the patellar tendon attachment to the tibia during puberty: a 12-month follow up in tennis players.Scand J Med Sci Sports. 2010;20:e35-e40.

10. De Flaviis L, Nessi R, Scaglione $P$, Balconi G, Albisetti W, Derchi LE. Ultrasonic diagnosis of Osgood-Schlatter and Sinding LarsenJohansson diseases of the knee.SkeletalRadiol. 1989;18:193-197.

11. Holik mf. Vitamin d status: measurement, interpretation, and, clinical application. Ann epidemiology. 2009;19(2): 73-78.

12. Demirag B, Ozturk C, Yazici Z, Sarisozen B. The pathophysiology of OsgoodSchlatter disease: a magnetic resonance investigation. $J \quad$ PediatrOrthop $B$. 2004;13(6):379-82.

13. Kaya DO, Toprak U, Baltaci G, Yosmaoglu B, Ozer H. Long-term functional and sonographic outcomes in Osgood-Schlatter disease. Knee Surg Sports TraumatolArthrosc. 2013;21:11311139.
14. Lanning P, Heikkinen E. Ultrasonic features of the Osgood-Schlatter lesion. J PediatrOrthop. 1991;11:538-540.

15. Vreju F, Ciurea P, Rosu A. Osgood-Schlatter disease - ultrasonographic diagnostic. Med Ultrason. 2010;12:336-339.

16. Nakase J, Goshima K, Numata H, Oshima T, Takata Y, Tsuchiya H. Precise risk factors for Osgood-Schlatter disease. Arch Orthop Trauma Surg. 2015 Sep. 135 (9):1277-81.

17. Benjamin M, Kumai T, Milz S, Boszczyk BM, Boszczyk AA, Ralphs JR. The skeletal attachment of tendons-tendon "entheses". Comp BiochemPhysiol A MolIntegr Physiol. 2002;133(4):931-945.

18. Benjamin M, McGonagle D. Entheses: tendon and ligament attachment sites. Scand J Med Sci Sports. 2009;19(4):520527. 19. Benjamin M, Kaiser E, Milz S. Structure-function relationships in tendons: a review. J Anat. 2008;212(3): 211-228.

19. Benjamin M, Toumi H, Suzuki D, Hayashi $\mathrm{K}$, McGonagle D. Evidence for a distinctive pattern of bone formation in enthesophytes. Ann Rheum Dis. 2009;68(6):1003-1010.

20. Blankstein A, Cohen I, Heim M, et al. Ultrasonography as a diagnostic modality in Osgood-Schlatter disease. A clinical study and review of the literature. Arch Orthop Trauma Surg 2001; 121: 536-539. 